\title{
A Cognitive Radio Receiver Supporting Wide-Band Sensing*
}

\author{
Volker BLASCHKE, Tobias RENK, Friedrich K. JONDRAL \\ Institut für Nachrichtentechnik, Universität Karlsruhe (TH), Karlsruhe, Germany \\ E-mail: \{blaschke,renk, fj\}@int.uni-karlsruhe.de \\ Received April 29, 2009; revised May 8, 2009; accepted May 10, 2009
}

\begin{abstract}
The specification of IEEE 802.22 defines the world-wide first cognitive radio (CR) standard. Within a range of $40 \mathrm{MHz}$ to $910 \mathrm{MHz}$ CR systems are allowed to allocate spectrum besides the currently established radio services like radio and TV broadcasting. In order to fulfill the regulative guidelines of interference limitations, a capable spectral sensing and user detection has to be provided. Due to the wide frequency range specified in IEEE 802.22 and the high dynamic range of signals allocated in this band there are high demands on the CR receiver's front-end. Especially the performance requirements on analog-to-digital converters increase significantly compared to current wireless systems. Based on measurements taken in this frequency range requirements to CR's ADCs are figured out. Furthermore, the measurement results are analyzed regarding expectable allocation scenarios and their impacts to spectral sensing. Derived from this results and a comparison of general spectral sensing mechanisms an approach for a CR receiver supporting wide-band sensing is presented. Considering the apriori information resulting from scenario analysis and including adapted information processing in the terminal the ADC's performance requirements can be reduced.
\end{abstract}

Keywords: Cognitive Radio, IEEE 802.22, Spectrum Sensing, A/D Conversion

\section{Introduction}

The term Spectrum Sensing becomes more and more important, especially in the context of cognitive radio (CR). Due to the increased request for wireless transmission resources and the ongoing installation of new radio access technologies for broadband access, enhanced research in the field of mobile CR receivers is necessary. Based on the results of spectral measurements $[1,2]$ a low utilization over wide frequency ranges was identified. This additionally motivates the development of intelligent radio resource allocation mechanisms to overcome this waste of resources. For increasing the overall utilization, dynamic allocation of free spectral resources that considers both the users and the spectral environment is required. This approach is supported by the CR concept $[3,4]$. Providing a dynamic resource allocation, sufficient information about the spectral environment has to be collected. Thereby, different acquisition methods

\footnotetext{
*This paper is an expanded version of the correspondent article accepted in the proceedings of "2008 IEEE International Conference on Communications Workshop."
}

can be used. On one side, all information are collected by a central control unit and distributed to simple mobile terminals. In this case traffic load information could be exchanged between joint networks via backbone [5]. Other approaches base on distributed sensing using all mobile terminals of a radio access network. This requires sensing capabilities in each mobile entity and an efficient algorithm for consolidation of the results. Especially in such scenarios swarm-intelligence algorithms could offer additional benefits. Nevertheless, an appropriate spectral sensing and information extraction forms a precondition for dynamic and efficient allocation mechanisms. In order to avoid unacceptable interferences a reliable detection of other users has to be supported by the CR. Therefore, both, the temporal as well as the spectral characteristics of the observed frequency band have to be known to the terminal. All these requirements lead to high demands on the radio's frontend.

In this paper the performance demands on analog-todigital converters (ADCs) in mobile CR receivers supporting spectral sensing are discussed. Based on the frequency ranges specified in IEEE 802.22 [6] general as- 
pects of wide-band sensing as well as specific demands on the structure of mobile CRs are presented. Analyzing the frequency range of IEEE 802.22 several types of channel utilization can be figured out. This a-priori knowledge combined with suitable information processing in the terminal will lead to an optimized front-end structure supporting wide-band spectral sensing.

The paper is structured as follows: In the next section a brief introduction to ADCs is presented. In Section 3, a detailed description of sensing algorithms in CR terminals is given. Considering the frequency bands specified in IEEE 802.22 the expected signal characteristics are identified. Based on this, two general wide-band sensing methods including a comparison of their demands to ADC's performance are described in Section 4. In Section 5 a $\mathrm{CR}$ receiver structure is presented including adapted spectral sensing combined with a convenient information processing. Finally, a conclusion is given.

\section{Analog-to-Digital Conversion in CR Receiver}

The digitization of the received signal is a basic component in each digital receiver. Only a suitable sampling and quantization of the analog input signal enables the receiver to provide the communication tasks supported by digital signal processing. Most CR concepts described in literature assume an appropriate ADC as precondition. But an efficient analog-to-digital conversion contains a lot of challenges in order to support the performance constraints assumed in these CR concepts.

Generally, the key parameters for summarizing the ADC's performance are stated resolution, signal to noise ratio, spurious free dynamic range, and power dissipation [7]. Furthermore, aperture jitter as well as two-tone intermodulation distortion is important for characterizing ADCs. A detailed description and performance analyzes can be found in [8] and [9]. In [8] the performance of on-the-market ADCs is analyzed in order to describe the evolution and trends in ADC's technology. This evaluation was continued in [9] including also present-day trends.

In this article ADC parameters which restrict an implementation of current ADCs in mobile CR terminals are figured out. These are sampling frequency $f_{\mathrm{s}}$, affecting the effective resolution bandwidth, effective number of bits $N_{\text {eff }}$, describing the dynamic range supported by the ADC, and power dissemination $P_{\text {diss }}$ resulting in battery running time.

In order to fulfill the Nyquist criterion the converter's sampling frequency $f_{\mathrm{s}}$ has to be more than two times the effective analog bandwidth [10]. Furthermore, the effective number of bits $N_{\text {eff }}$ is lower than the stated number of resolution bits specified by the vendor. Due to hardware imperfections and quantization noise $N_{\text {eff }}$ is [8]

$$
N_{\text {eff }}=\frac{\mathrm{D}-1.76}{6.02}
$$

where $\mathrm{D}$ describes the effective dynamic range of the converter in $\mathrm{dB}$. Especially for detection and sensing applications a high dynamic range is of increased importance. If the dynamic range of the expected input signals is higher than ADC's dynamic range, weak signals may not be detected due to resolution limitations.

Having a look to the results depicted in [8] and [9] three main hardware architecture concepts become potential candidates for implementation on mobile CR terminals. Flash converters offer sampling rates of about 1 Gsps due to a parallel comparator structure. But this requires high hardware effort which causes increased power dissemination. Therefore, this architecture is unattractive to mobile applications. Due to the high hardware effort an effective resolution of only $N_{\text {eff }}=6 \ldots 8$ Bits can be supported. Using Pipelined ADCs $N_{\text {eff }}$ can be increased up to 15 Bits but the sampling frequency $f_{\mathrm{s}}$ is less than 500 Msps. Due to the specific design, implementation of analog track-and-hold blocks is required. The third group of potential candidates is $\Sigma \Delta$-converters. The available effective resolution is up to $20 \mathrm{Bit}$ but the available sampling rate is less than $100 \mathrm{Msps}$. Though, due to their low power consumption these ADCs are very interesting for an implementation in mobile $C R$ terminals. A detailed description of the different ADC structures can be found in $[10,11]$.

\section{Spectrum Sensing and Related Hardware Impacts}

Sensing the current channel state is one important task of each radio system using dynamic channel allocation. The well-known IEEE 802.11 wireless LAN systems, for instance, use carrier sense multiple access with collision avoidance (CSMA/CA). This multiple channel access scheme requires sensing and detection of channel allocation. In such systems, sensing bandwidth and transmission channel bandwidth are the same.

During the last years several approaches for dynamic spectrum allocation (DSA) [12] and channel allocation adapted to the current spectrum situation and user requirements were published [5]. These concepts combine available transmission resources of several systems in order to optimize the spectrum utilization of all considered systems. This would require the availability of the current system state to all other systems. Assuming a coupling of combined systems and a general control entity, the information exchange can be realized using traffic control channels in the wired backbone network. So, each system can handle and optimize the channel allocation of its subscribers considering this additional traffic 
load information. Inter-system handover need to be initiated and controlled by the general control entity. Basically, this architecture requires cooperation between all radio access networks participating in DSA, which again results in a relatively static system configuration.

Another approach for increasing the spectral utilization is overlay systems [13]. Based on the fact that wide frequency ranges offer a lot of unallocated transmission capacity, this approach describes the allocation of local networks exploiting temporarily and/or locally unoccupied transmission channels. Due to the basic precondition that licensed systems should not be changed for overlay usage, the rental user must observe the communication channel in order to provide a reliable detection of the licensed user's channel allocation.

Furthermore, the rental user's signal must be adapted to the licensed system's transmission parameters regarding channel bandwidth, maximum channel allocation duration, transmission power, etc. Static system parameters, e.g., channel bandwidth, can be defined in a database available for each rental user. But for dynamic parameters, e.g., current channel allocation, licensed user's allocation statistics have to be observed and analyzed at present. So, a continuous spectral observation has to be done by the overlay system. In order to get information about the allocation of frequency bands, energy detection can be used. Comparing the received signal power with the noise level general channel allocation information can be collected. If the signal power is higher than the measured noise level the channel is already occupied. Especially, in case of weak signals that are close to noise level the power detection can fail. So, analyzing signal's higher order statistics or other feature detectors may overcome this drawback [14].

Having a look to the IEEE 802.22 specification, the communication channel bandwidth is $B_{\mathrm{ch}}=6 \ldots 8 \mathrm{MHz}$ and the frequency range specified for allocation is between $41 \mathrm{MHz}$ and $910 \mathrm{MHz}$ with respect to national regulations [6]. So, the overall system bandwidth, that has to be observed, is $B_{\mathrm{S}}=869 \mathrm{MHz}$. This is more the 100 times the signal's bandwidth $B_{\mathrm{ch}}$. In order to provide a flexible CR system which is able to optimize spectral utilization, the full frequency range has to be supported by mobile terminals. This also includes the ability for a fast and efficient sensing of wide ranges in order to adapt transmission parameters to the licensed user's allocation. Supporting high signal bandwidth directly affects the hardware architecture of a terminal. Especially the analog signal processing and the ADC limit the supported bandwidth. As it is described in Section 2, there is a trade-off between the signal's bandwidth and the dynamic range of the converter.

Having a look to the receiving signals within this frequency range, several allocation characteristics can be pointed out. In Figure 1 the received signal power per frequency averaged over a sensing period of $3 \mathrm{~h}$ is depicted. The frequency range of $f=41 \ldots 910 \mathrm{MHz}$ represents the overall range specified for IEEE 802.22. As it can easily be seen, there are wide frequency ranges where a very low averaged signal energy is detected. But also high utilized bands can be pointed out. Between 88 $\mathrm{MHz}$ and $108 \mathrm{MHz}$ the European FM radio broadcast service is allocated (cf. ch 1). Furthermore, some TV broadcast signals as well as temporarily allocated channels can be noticed. For describing the channel utilization during sensing time a binary spectrogram can be defined:

$$
\left.O\{x(t)\}\right|_{f=f_{m}}=\left\{\begin{array}{lll}
1 & \text { for } & \left.S\{x(t)\}\right|_{f=f_{m}}>P_{\text {th }} \\
0 & \text { for } & \left.S\{x(t)\}\right|_{f=f_{m}} \leq P_{\mathrm{th}}
\end{array},\right.
$$

where $x(t)$ is the received signal, $\left.S\{x(t)\}\right|_{f=f m}$ describes the spectrogram of $x(t)$ at frequency $f_{m}$, and $P_{\text {th }}$ describes the detection threshold. The resulting binary description of the spectral allocation can be averaged over sensing time using a window length $N_{w}$. So, the averaged channel utilization can be written as

$$
\left.\bar{O}_{t_{n}}\right|_{f=f_{m}}=\left.\frac{1}{N_{w}} \sum_{t=t_{n}}^{t_{n}+N_{w}+1} O_{t_{n}}\right|_{f=f_{m}},
$$

where $t_{n}$ is the time index. The resulting characteristics for three different frequencies are depicted in Figure 2. The curve of channel 1 describes a typical broadcast channel utilization. Channel 2 offers a varying averaged occupation between 0.01 and 0.92 . In channel 3 an averaged utilization of 0 can be noticed. During this period the channel is not used by the licensed user and would be interesting for $\mathrm{CR}$ resource allocation. In order to detect

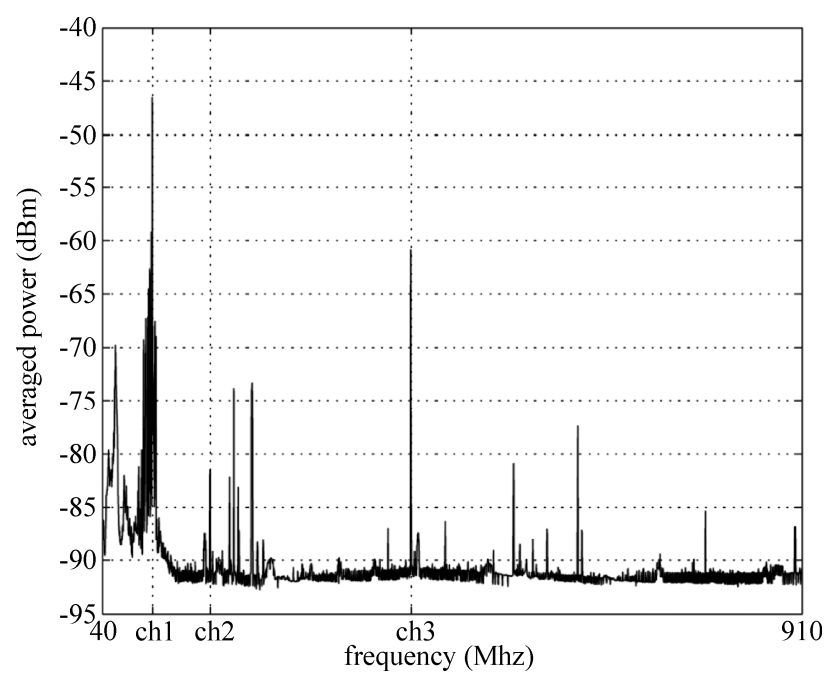

Figure 1. Averaged power vs. Frequency in IEEE 802.22 frequency range. 


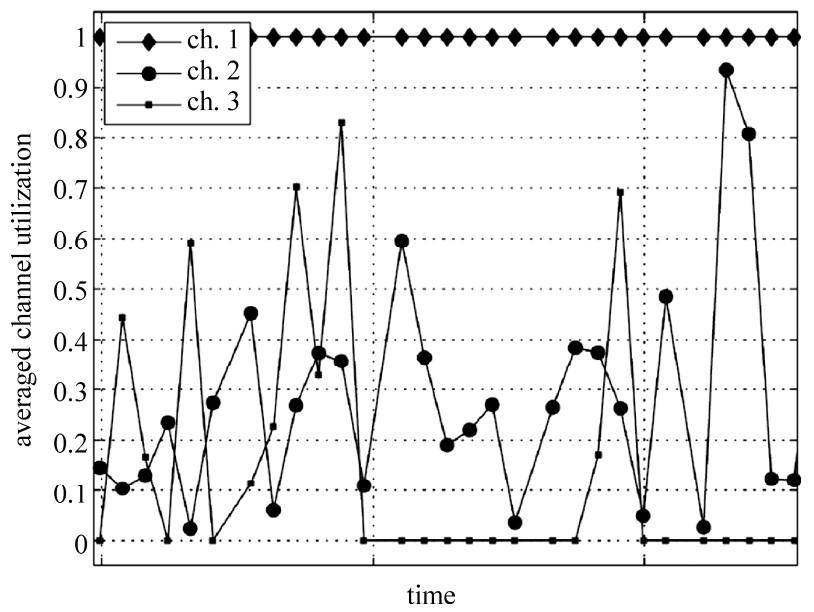

Figure 2. Averaged channel utilization of channel 1, 2 and 3.

such periods that are also called white spaces [4] a suitable observation of wide frequency ranges has to be provided. As it can be noticed in Figure 1, high differences in the dynamic range of the depicted signals has to be considered. During measurements a maximum signal power of $-41.8 \mathrm{dBm}$ at channel 1 was observed. The general noise level is $-92 \mathrm{dBm}$ measured in unallocated sub-bands. So, the overall dynamic range compared to the bandwidth of more than $850 \mathrm{MHz}$ marks the main challenge in finding suitable solutions for mobile CR receivers supporting this wide frequency range. In the next section two general sensing methods supporting these requirements will be described.

\section{Spectrum Sensing Methods}

Several methods can be used for analyzing wide ranges of radio spectrum. The two general concepts, sweeping a small detection window over the observed frequency band and wide band analog to digital conversion followed by energy or feature detection are briefly de- scribed in the following subsections. Their ability for implementation in a mobile $\mathrm{CR}$ receiver will also be discussed.

\subsection{Basics on Spectral Analysis}

Due to the fact that currently available ADCs possess a limited bandwidth, it is not possible to digitize large frequency spans at once. Therefore, so-called fast Fourier transform (FFT) analyzers are only usable for low frequency signals. In order to investigate high frequency signals, superheterodyne receivers must be applied. Here, the overall input frequency span is mixed to a common intermediate frequency (IF) by a tunable local oscillator [15]. Spectral resolution is directly determined by the IF filter. The smaller the resolution bandwidth $B_{\mathrm{R}}$, the higher the spectral resolution. A well-known problem occurs if the input frequency range is more than two times bigger than the IF, because then suppression of the image frequency is not possible without affecting the input signal. Hence, a tunable bandpass is necessary. This problem can be overcome if several IF stages are used, where the first one transforms the input signal to a higher frequency. It is then possible to suppress the image frequency without affecting the input signal.

Figure 3 shows a superheterodyne receiver with two IF chains for the IEEE 802.22 specification. The RF input signal first passes RF attenuation that helps to prevent overload and distortion. Afterwards, a preselector lowpass filters out higher frequency signals. In order to mix the RF signal up to $1000 \mathrm{MHz}\left(f_{\mathrm{IF} 1}\right)$, the first local oscillator (LO 1) must operate in a frequency range from $1041 \mathrm{MHz}$ to $1910 \mathrm{MHz}$. This leads to an image frequency that ranges from $2041 \mathrm{MHz}$ to $2910 \mathrm{MHz}$ which can easily be suppressed by the following IF 1 filter. After this, a second local oscillator (LO 2) with a frequency of $970 \mathrm{MHz}$ mixes the $1000 \mathrm{MHz}$ signal down to $f_{\mathrm{IF} 2}=30 \mathrm{MHz}$. Image frequency is $940 \mathrm{MHz}$ which

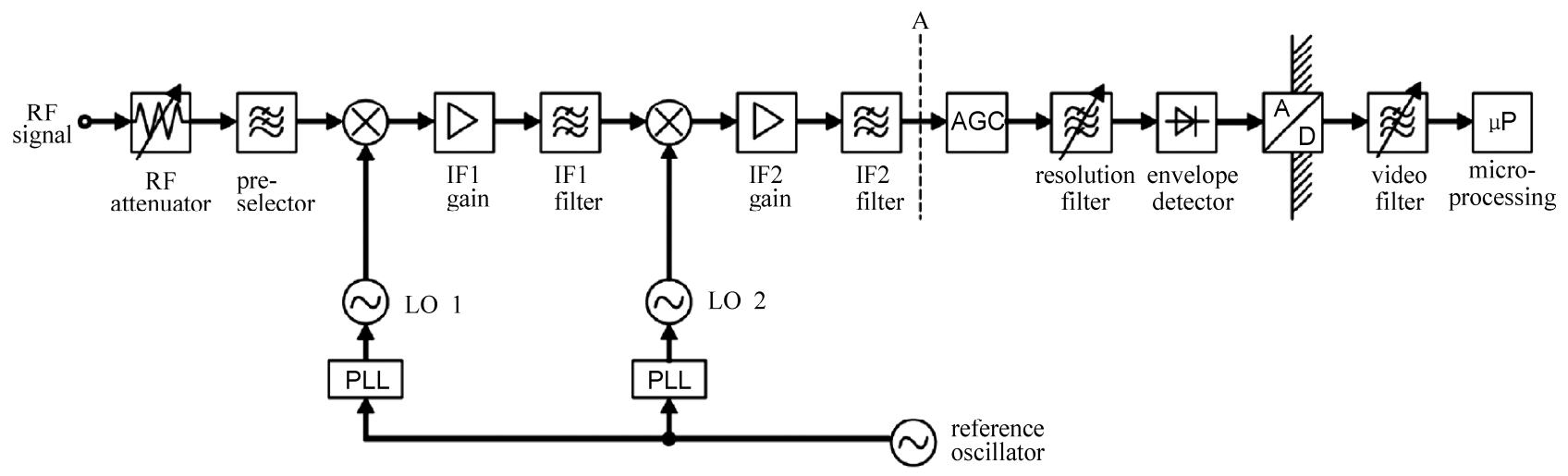

Figure 3. Superheterodyne receiver with two IF chains and low frequency ADC fort he IEEE 802.22 specification. 
again can easily be filtered out by the IF 2 filter. Both LOs are controlled by PLLs that are connected to a reference oscillator to increase frequency accuracy. The automatic gain control (AGC) block is followed by a tunable bandpass filter that determines the resolution bandwidth. The ideal case for the resolution filter is a rectangular filter with bandwidth $B_{\mathrm{R}}$. In order to achieve short measurement times, however, optimized Gaussian filters are used that are temperature stable and possess a higher bandwidth accuracy as well. Nevertheless, $B_{R}$ influences the sweep time $T_{\mathrm{sw}}$ that is necessary to scan the whole frequency range $B_{\mathrm{S}}$. If measurement time falls below $T_{\text {sw }}$, amplitude losses and signal distortions occur that eventually lead to frequency offsets. Subsequent to filtering is the envelope detector and ADC. The video filter is a lowpass that suppresses noise and helps to smooth the signal spectrum. The video bandwidth $B_{\mathrm{V}}$ acts inversely proportional to the sweep time $T_{\mathrm{sw}}$. The micro-processing block $(\mu \mathrm{P})$ includes, e.g., averaging and threshold decision making.

For the definition of the required sweep time, two cases must be taken into consideration, one where the video bandwidth is higher than the resolution bandwidth and vice versa [15]:

$$
T_{\mathrm{sw}}=\left\{\begin{array}{c}
k \cdot \frac{B_{\mathrm{S}}}{B_{\mathrm{R}}^{2}} \text { for } B_{\mathrm{V}}>B_{\mathrm{R}} \\
k \cdot \frac{B_{\mathrm{S}}}{B_{\mathrm{R}} \cdot B_{\mathrm{V}}} \text { for } B_{\mathrm{V}}<B_{\mathrm{R}}
\end{array},\right.
$$

where the parameter $k$ denotes a proportional factor that is usually in the range of 1 to 3 . The first case of Equation (4) is illustrated in Figure 4. System parameters were chosen according to the IEEE 802.22 specification. The vertical dashed lines define the channel bandwidth of $B_{\mathrm{ch}}=6 \ldots 8 \mathrm{MHz}$ [16]. It can easily be seen that for one

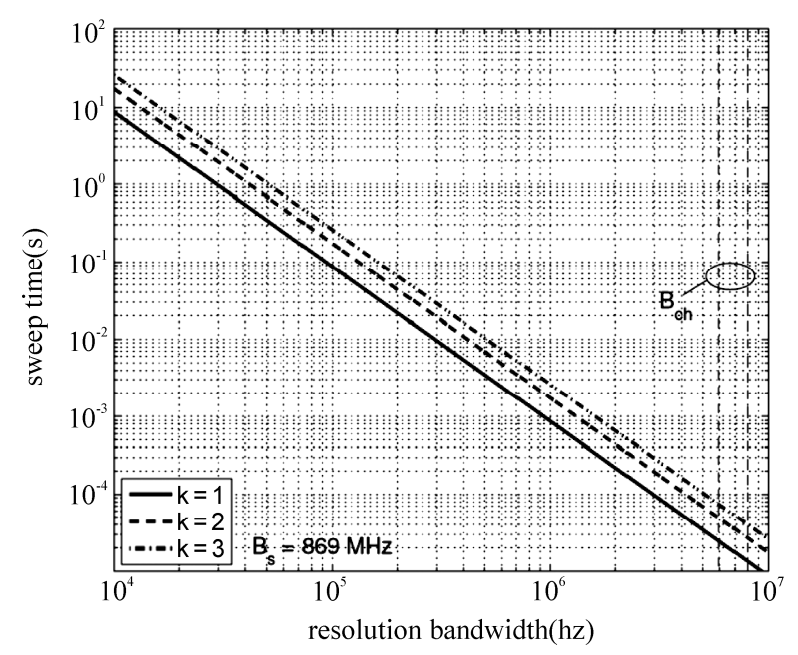

Figure 4. Sweep time $T_{\mathrm{sw}}$ in relation to resolution bandwidth $B_{R}$ with parameter $k=1,2,3$. single scan through the whole frequency range of 869 $\mathrm{MHz}$ approximately $310^{-4} \mathrm{~s}$ are necessary if we consider a resolution bandwidth of $B_{\mathrm{R}}=2.5 \mathrm{MHz}$. (This means that we take two to four frequency bins per channel.)

As it can be seen in Figure 3, the ADC is located after the resolution filter and the envelope detector. This means that only a bandwidth of $B_{\mathrm{R}}$ has to be digitized which leads to a higher amplitude resolution. Moreover, a quite simple ADC is sufficient in that case. Drawbacks are, however, that pretty much analog hardware is necessary and that the measurement time is increased (cf. Section 5). Additionally, no feature detection is possible since only energy detection is performed.

\subsection{Wide-Band Sensing}

Another possibility for energy detection is the direct conversion of the wide-band input signal. This reduces the number of intermediate frequency stages required for sweeping the detection window but significantly increases the performance requirements at the ADC.

Ideally, the incoming analog signal is bandpass filtered by a bandpass with the system bandwidth $B_{\mathrm{S}}$. Afterwards, the signal is amplified and down converted from the radio frequency $f_{\mathrm{RF}}$ to an intermediate frequency $f_{\mathrm{IF}}$. Subsequent to a second filtering and AGC, the analog to digital conversion is done. The following data processing includes an FFT in order to extract the current power allocation over the frequency.

After digitization the signal contains information of the complete observed frequency range. Of course, the information depth is characterized by the resolution performance of the ADC, which is a main drawback of this approach. As it was shown in the section before, the observed signal is characterized by a high variation of the spectral power density. The overall dynamic range is more than $50 \mathrm{~dB}$. Furthermore, under-utilized small-band signals with weak signal amplitudes can be noticed. In order to provide a reliable detection of the licensed user, these signals still have to be noticeable after the ADC, otherwise the signal detection fails. Therefore, the dynamic range of the ADC is an important parameter for CR terminals.

Based on Equation (1) the presented measurements would require a minimum resolution of $N_{\text {eff }}=9$ Bits. Besides a high bit resolution, also a high sampling rate is required for a wide band digitization. Following the example of IEEE 802.22, the sampling rate is $f_{\text {samp }}=$ 1.82 Gsps meeting the Nyquist criterion. Due to the overall frequency range from $41 \mathrm{MHz}$ up to $910 \mathrm{MHz}$, a bandpass sub-sampling cannot be used for reduction of $f_{\text {samp. }}$.

As stated in [9], the maximum sampling rate for an effective resolution of $N_{\text {eff }}=9$ Bits is about $f_{\text {samp }}=500$ Msps ... 1 Gsps. The analysis in [8] and [9] also figure 
out those sampling rates significantly higher than 100 Msps can only be handled by Flash or Pipelined converters. As described in Section 2, these two architectures are characterized by a high power consumption and, therefore, not preferable for an implementation in mobile terminals. The group of $\Sigma \Delta$-converters offer lower power consumption but cannot provide the high sampling rate available for Flash converter. In order to suppress the undesired signals also analog notch filter can be applied. This requires a first scan for in identification of the strongest signals. After tuning the notch filter to these frequencies a second scan is used for detection of weak signals. Besides doubling the scan time also the analog hardware effort increases significantly.

Compared to the energy detection described above, a wide band digitization offers a better time resolution in a wide frequency band. Thus, a detailed extraction of temporal features is possible. A high time resolution becomes important for detection of very short channel allocations or for detailed analyzing of the licensed user's allocation statistics.

Both concepts offer different advantages which are required for a successful operation of flexible overlay systems. On the other side, each concept has significant drawbacks that can not be solved within the next couple of years and preclude an implementation in mobile terminals. So, a combination of both could give the opportunity to combine the advantages. This approach is discussed in Section 5.

\section{Wide-Band CR Receiver for IEEE 802.22}

\subsection{Sub-Band Spectrum Sensing}

As we presented above, a digitization of the complete system bandwidth $B_{\mathrm{S}}$ is not useful regarding to technical and economical constraints. On the other side, the extension of spectral sensing described for CRs will support an increased spectral utilization. In order to simplify the spectrum sensing and to reduce the hardware requirements described in Subsection 4.1., we will analyze the measurement results with respect to signal characteristics expectable in the IEEE 802.22 frequency range. Having a closer look to the results depicted in Figure 1 and Figure 2, it can be seen that channel utilization in the sub-band allocated by broadcast services (cf. ch 1) does not change during the measurement time. Furthermore, the received signal power of broadcast transmitters averaged over the sensing time is significantly higher than the noise level. In general, the average power level of the broadcast signals is higher than $-70 \mathrm{dBm}$ and even higher than $-50 \mathrm{dBm}$ considering the strongest signal. Therefore, a continuous sensing of these frequency ranges does not offer any additional information for an operating $\mathrm{CR}$ system. This leads to the opportunity to exclude such quasi-static frequency ranges considering its information entropy during the spectrum sensing phase. Furthermore, a decreased dynamic range of the input signal reduces the ADC's hardware recommendations. Without loss of generality statistical independence of observed communication channels can be assumed. So, the spectrum that is observed for detection of averaged channel allocation can be divided into several sub-bands. These sub-bands do not need to be observed at the same time but can be sensed sequentially as long as the sensing is repeated periodically and the sensing interval as well as the sensing period is suitable to the licensed user's signal. Based on this knowledge and assuming a suitable sensing sequence the full system band can be split into $M$ sub-bands:

$$
B_{\mathrm{S}}=M \cdot B_{\text {sub }}
$$

Each sub-band is separately digitized reducing the sampling rate of the ADC. Due to decoupling highly utilized communication channels and frequency bands with low spectral utilization, the dynamic range of the ADC's input signal can be optimized as well, which results in an enhanced sensing of weak signals. Additionally, the suppression of strong signals using analog notch filter is not necessary, because the reduced sampling rate enables higher bit resolution.

\subsection{Receiver Structure}

The system architecture described in Subsection 4.1 defines the bases for our wide-band CR supporting subband sensing. In contrast to the structure depicted in Figure 3 the ADC is placed directly after the IF 2 filter. This position is marked with the letter ' $A$ '. The adapted signal processing of the wide-band $\mathrm{CR}$ receiver is depicted in Figure 5. Until the marker ' $A$ ' the analog signal processing is the same as described in Subsection 4.1. In order to support the IEEE 802.22 specifications the sub-band bandwidth is defined to $B_{\text {sub }}=50 \mathrm{MHz}$ including six sub-channels at a bandwidth of $8 \mathrm{MHz}$ up to eight sub-channels at a bandwidth of $6 \mathrm{MHz}$, respectively. Similar to the number of sweep points defined in analyzer detection the LO 1 can be tuned to 20 predefined frequency steps resulting in a small sub-band overlap. For a more flexible sub-band configuration, a continuous oscillator tuning could also be implemented. Generally, digitization of a $50 \mathrm{MHz}$ sub-band requires a sampling rate of about $100 \mathrm{Msps}$. In the following digital sensing processing energy detection as well as feature detection or other signal detection algorithms could be applied. In Figure 5 the block structure for energy detection is depicted. Due to the digital processing the single scan time 
can be reduced by factor $1 / 20$ compared to the analog processing using $B_{\mathrm{R}}=2.5 \mathrm{MHz}$.

Besides the reduction of scan time the proposed sub-band digitization offers the possibility of sensing adjacent channels of the currently allocated communication channel. As it is depicted in Figure 5 the received signal is used for communications and sensing processing in parallel. This means that all spectral information within the actual sub-band can be collected along with current data transmission. Furthermore, the additional time released by the parallel sensing and communication processing can be used for additional sensing of other sub-bands. The additionally obtained information increases the CR's knowledge about its spectral environment. But besides an efficient sensing algorithm, also a suitable information processing and knowledge storage has to be applied in mobile CR terminals. In the next subsection an algorithm is presented what bases on the proposed sub-band sensing.

\subsection{Information Processing}

Another question in the context of CRs deals with processing the data gained from spectrum sensing. As it was described by Mitola [3], one important enhancement of CRs compared to SDRs is the implementation of reasoning algorithms. Reasoning can be applied for user centric applications like user interface adaptation or providing user-specific local information and news services. Moreover, processing of experiences can also be used for optimizing the spectral sensing procedure. As long as a predefined performance can be fulfilled, it is not necessary to change the terminal's configuration or the allocated communication channel. Hence, sensing the full spectral range is not necessary for most of the time. In case that the currently occupied sub-band cannot longer be allocated by rental users the information of the next most suitable sub-band is required. Due to the possibility of a direct sub-band scanning described above the scanning procedure can be limited to such sub-bands which offered low channel utilization in the past. In order to get

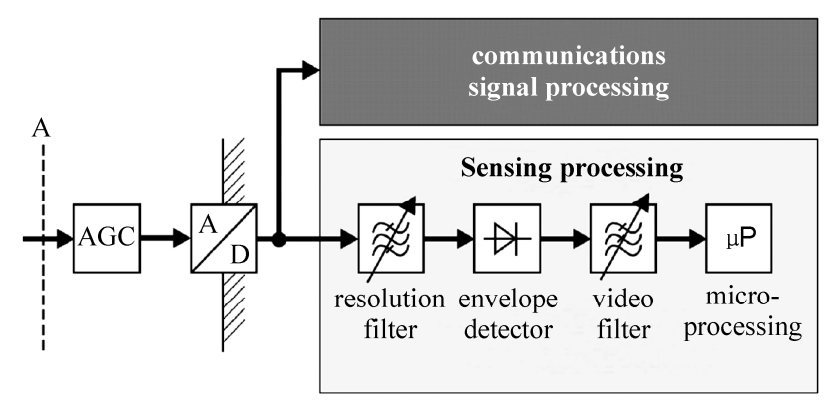

Figure 5. Structure of wide-band CR receiver supporting sub-band sensing. a first overview of a sub-band, the averaged utilization (cf. (3)) of a communication channel or a complete sub-band could be considered. Due to the digitization of a full sub-band $B_{\text {sub }}$, all communication channels in this band can be observed simultaneously. As long as the current sub-band can offer some free radio resources to the CR overlay system, other sub-bands need not be observed continuously. A periodical short scan provides information to approximately trace the sub-band utilization. Based on this sensing result, the sub-band can be ordered with respect to the current utilization. In case of shifting overlay users to another sub-band, the sub-band offering the lowest utilization can be observed in detail.

Since spectrum sensing is only one task of a CR terminal, besides radio communication, an intelligent scheduling of the sensing periods is necessary. In case of a feasible number of CR nodes at one location, the quality of the detection result will not increase significantly compared to the number of additional nodes. Thus, distributed sensing of different sub-bands that is provided by several nodes at the same time will help gain more information of the overall frequency range. In order to use this advantage, the challenge of collecting the information from all distributed nodes need to be solved.

In [13] an innovative approach for distributed sensing that provides a solution to overcome the hidden-node problem was proposed. In the described system all nodes sense the same sub-band synchronously. After the sensing, all binary detection results are collected at one master station of the local network. The detection results of each single channel within the observed sub-band are coded in a one bit decision. These bits are sent simultaneously by all nodes that are connected to the master station. For a detailed description of the algorithm the reader may be referred to [13]. Basically, the simultaneous transmission leads to a superposition of all detection results, which can be interpreted as a logical OR operation. Thus, a reliable detection of an increased area can be provided.

Adopting this approach to the problem of increasing the observed spectral range, we can use the following strategy: Besides the signaling of the detection results within the currently used sub-band another sensing and signaling period can be added. During this period an additional sensing and signaling of adjacent sub-bands may be executed. Following the signaling method described in [13] the sensing results are superposed. Thus, the calculated sub-band utilization is a rough estimation of the available resources. In case of a low utilization a detailed sensing will follow. The measurement process supporting such a distributed sensing is depicted in Figure 6. The data transmission including sensing and signaling regarding [13] in the currently used sub-band is named S1. During this phase dedicated nodes observe also adjacent sub-bands. These sensing results are sent to the 


\begin{tabular}{|l|l|l|l|l|l|l|l|l|l|l|l|}
\hline $\mathrm{S} 1$ & $\mathrm{~S} 2$ & $\mathrm{~S} 1$ & $\mathrm{~S} 2$ & $\mathrm{~S} 3$ & & $\mathrm{~S} 1$ \\
\hline
\end{tabular}
S1...State1;
S2...State2;
S3...State3

Figure 6. Sensing process for wide-band CRs supporting sub-band processing.

central control station during the phase $S 2$. Furthermore, a full range scan could be initiated, which is named $S 3$. Since the full scan needs, however, more time compared to the normal periodic sub-band scan, the communication in the CR system may be affected by the full scan. Due to the ranking of the sub-band utilization, only the most suitable sub-bands need to be considered. A full processing of allocation information is only required for the current sub-band. All other sub-bands are characterized by an averaged utilization index that reduces the memory and processing effort in the mobile terminal. For a detailed investigation of the proposed distributed sensing method, e.g., bio-inspired algorithms could be taken into consideration.

\section{Conclusions}

In order to increase spectral utilization future CR receivers have to provide spectrum sensing capabilities. Applying DSA mechanisms require suitable spectrum sensing capabilities in order to adapt the radio transmission to the identified spectral environment. In the CR standard IEEE 802.22 a frequency range from $41 \mathrm{MHz}$ to $910 \mathrm{MHz}$ is specified. Within this spectral band CR networks can be established under the limitation that the already established radio services are not interfered unacceptably. In order to provide a reliable signal sensing and detection in the CR terminals several preconditions to the receiver's front-end have to be fulfilled. In this paper demands on ADCs in such wide-band scenarios are discussed in detail. As presented in Section 2, the general performance of ADCs is characterized by the trade-off between supported bandwidth and dynamic range defined by the effective number of bits. Today's ADC support sampling rates up to several Gsps at the expense of low dynamic range and high power consumption. But the demand for a high sensing quality in mobile receivers leads to the contrary request for high dynamic ranges at a low power consumption. Thus, the input bandwidth has to be reduced. In Section 3 the different radio services allocated in the considered frequency range are analyzed. It is shown, that the utilization varies significantly over the frequency. Thus, different demand for sensing in the sub-bands can be observed. Especially sub-bands allocated by broadcast radio services do not offer additional radio transmission resources but increase the demands on ADC's performance significantly. Based on the sensing algorithms described in Section 4, a receiver for wide-band mobile CRs based on the superheterodyne principle is presented in Section 5. Due to the proposed distribution of the wide-band into 20 sub-bands high-utilized sub-bands can be masked while sensing of under-utilized sub-bands benefits from the increased resolution of the ADC resulting from the decreased input signal bandwidth. It combines this sub-band sensing method with the presented information processing results in a capable mobile receiver structure for IEEE 802.22 CR networks.

\section{References}

[1] FCC, "Spectrum policy task force report, ET Docket No. 02-155," Technical Report Series, November 2002.

[2] Shared Spectrum Company, "Comprehensive spectrum occupancy measurements over six different locations," August 2005,

http://www.sharedspectrum.com/.

[3] J. Mitola, "Cognitive radio-an integrated agent architecture for software defined radio," Ph.D. dissertation, Royal Institute of Technology (KTH), Kista, Sweden, 2000.

[4] S. Haykin, "Cognitive radio: Brain-empowered wireless communications," Selected Areas in Communications, IEEE Journal on, Vol. 23, No. 2, pp. 201-220, February 2005.

[5] P. Cordier et al., "E2R cognitive pilot channel concept," IST Mobile Summit, Mykonos, Greece, June 2006.

[6] IEEE, "P802.22: Cognitive radio, wide regional area network," Technical Specifications, May 2005.

[7] R. H. Walden, "Analog-to-digital converter technology comparison," IEEE GaAs IC Symposium Technical Digest, pp. 217-219, October 1994.

[8] R. H. Walden, "Analog-to-digital converter survey and analysis," Selected Areas in Communications, IEEE Journal on, Vol. 17, No. 4, pp. 539-550, April 1999.

[9] B. Le, T. W. Rondeau, J. H. Reed, and C. W. Bostian, "Analog-to-digital converters," Signal Processing Magazine, IEEE, Vol. 22, No. 6, pp. 69-77, November 2005.

[10] R. Plassche, CMOS Integrated Analog-to-digital and Digital-to-analog Converters, 2nd Edition, Kluwer Academic Publishers, Boston/ Dordrecht/London, 2003.

[11] B. Brannon, Software Defined Radio-Enabling Technology, John Wiley and Sons, London, W. Tuttlebee, Ed., ch. 
Data Conversion in Software Defined Radios, pp. 99-126. 2002.

[12] P. Leaves et al., "A summary of dynamic spectrum allocation results from drive," in IST Mobile and Wireless Telecommunications Summit, pp. 245-250, June 2002.

[13] T. A. Weiss and F. Jondral, "Spectrum pooling: An innovative strategy for the enhancement of spectrum efficiency," Communications Magazine, IEEE, Vol. 42, No. 3, pp. 8-14, March 2004.

[14] D. Cabric et al., "Implementation issues in spectrum sensing for cognitive radios," in Signals, Systems and Computers, 2004, Conference Record of the ThirtyEighth Asilomar Conference on, Vol. 1, pp. 772-776, November 2004.

[15] C. Rauscher, Grundlagen der Spektrumanalyse, Rohde \& Schwarz, 2004.

[16] C. Cordeiro et al., "IEEE 802.22: An introduction to the first wireless standard based on cognitive radios," Journal of Communications, Vol. 1, No. 1, April 2006. 